\title{
Factors Influencing Utilisation of Diagnostic Counselling and Testing for HIV among Tuberculosis Patients at Monze Mission Hospital, Zambia
}

\author{
Mulenga $J^{1,}$ Mwape $L^{2}$ and Mukwato $P^{3}$ \\ ${ }^{1}$ Monze Schools of Nursing and Midwifery Box 660029, Monze \\ ${ }^{2}$ Department of Nursing Sciences, School of Medicine, University of Zambia, Lusaka, Zambia, \\ ${ }^{3}$ Assistant Dean Undergraduate, School of Medicine, University of Zambia, Lusaka, Zambia \\ Corresponding Author: \\ Corresponding Author: Joyce Mulenga. Department of Nursing Sciences, School of Medicine, \\ UNZA, Box 50110, Lusaka, Zambia. Email: Joycemulenga2009@yahoo.com
}

\begin{abstract}
Background: Tuberculosis (TB) and human immunodeficiency virus (HIV) constitute the main burden of infectious disease in resource-limited Countries $^{6}$. Persons infected with HIV are particularly susceptible to tuberculosis, both from the reactivation of latent infection and from new infection with rapid progression to active disease ${ }^{8}$. An HIV-positive individual has ten times increased risk of developing TB compared to an HIV-negative person the lifetime risk is 50\% for an HIV-positive person and 5-10 percent for an HIV-negative ${ }^{9}$. Estimates by the World Health Organization (WHO) indicate that there are more than 9 million new active TB cases, close to 2 million deaths per year, 2.6 million new cases of HIV infection, and 1.8 million AIDS-related deaths occur per year ${ }^{6}$.
\end{abstract}

Main objective: To explore factors influencing utilisation of Diagnostic Counselling and Testing for HIV among TB patients.

Methods: A hospital-based cross-sectional study was carried out to assess the utilisation of Diagnostic Testing and Counselling for Human Immune Virus/Acquired Immune Deficiency among tuberculosis patients. Zambia. A non-experimental, which is exploratory study design, was used in this study. The study employed a quantitative approach because little is known about DCT utilisation.

Results: The level of knowledge and confidentiality were statistically associated with DCT.Overall $(n=226)$, majority $150(66.3 \%)$ of the patients did not utilise diagnostic counselling and testing services while $76(33.6 \%)$ utilised diagnostic counselling and testing. The odds ratio of confidentiality was OR $0.52-1.637$ and P-value 0.050 while the level of knowledge; OR was 0.719-45.785, and P-value was 0.048 , and the variables were sta- tistically significantly associated with Diagnostic Counselling and Testing. The study showed that the patients who did not utilise DCT had 47\% decrease in the odds of achieving a high level of knowledge and confidentiality were less than 0.05 and therefore failed to reject the null hypothesis. Multivariate binary logistic regression model predicted that confidentiality and knowledge were associated with DCT at $p$-value 0.05 .

Conclusion: Diagnostic Counselling and Testing utilisation were low in the study population. Level of knowledge and confidentiality were the factors found to be associated with diagnostic testing and counselling. The findings suggest the need for information, education and communication as patients lack information on the importance of tuberculosis patients taking up the test.

Keywords: Utilisation, Uptake, Diagnostic testing and counselling, HIV, Tuberculosis.

\section{INTRODUCTION}

The co-infection between HIV and TB has become a public health concern. More than 40 years after launching the first TB control programme in Zambia. Seventy percent of identified TB patients are co-infected with HIV making it critical to improve the diagnosis and treatment. The realisation that it will be impossible to curb the spread of HIV and AIDS without tackling TB and vice versa has led to an upsurge in research, surveillance and treatment initiatives into the two diseases that are feeding off each other to devastating effect.

Globally, TB remains a health problem of vast dimensions affecting millions of people each year. TB is a leading cause of morbidity and mortality among people living with HIV/AIDS (Peter et 
al; 2000). The HIV pandemic presents a massive challenge to the control of tuberculosis. Out of an estimated 8.3 million TB cases diagnosed worldwide, about 12 percent attributable to HIV (WHO, 2004. Although HIV infection impacts the tuberculosis epidemic, TB programmes have focused on TB case finding and treatment, with little attention to HIV/AIDS interventions. Tuberculosis is the leading cause of HIV related morbidity and mortality because little attention has been paid to TB (Peter, 2002). According to David, (2004), untreated HIV infection leads to increased susceptibility to infections, including TB. In India alone, about 2.5 million people are currently infected with HIV, of whom $40 \%$ are also co-infected with TB (Ghiya et al 2009). TB infection contributes to a further reduction in CD4 cell count accelerating the progression of HIV infection to Acquired Immunodeficiency Syndrome (AIDS) increasing the mortality and morbidity (Wanchu et al 2010). A study done in 2010 in Pakistan found that only 2\% of TB patients knew their HIV status, and nationally, only 22TB/ HIV patients were reported (WHO, 2011).

Regionally, Sub-Saharan Africa remains the global region most affected by HIV and AIDS; more than two-thirds of all the people with HIV live in Sub-Saharan Africa (WHO, 2014 Tuberculosis (TB) incidence is increasing in much of sub-Saharan Africa, largely due to the high prevalence of HIV infection (WHO, 2006). According to WHO (2007), findings reveal that TB was responsible for 1.3 million deaths among HIV negative incident cases in 2003. In addition, it was the most common cause of mortality among HIV patients in sub-Saharan Africa (Moore et al, 2007). The HIV prevalence among tuberculosis patients ranged from 20 to 60 percent between 1995 and 2005 (Corbett, 2006). This has led to a consensus that all TB patients should be tested for HIV infection in order to offer ART services where a patient is found positive (Srikantiah, 2007). The current HIV testing services trend remains poor in low and middle-income countries, including setting in which HIV counselling and testing are routinely offered. The number of people who utilise DCT services remains low in many countries.

Zambia has one of the world's most devastating HIV and AIDS epidemics in Southern Africa, and therefore, DCT is increasingly being recommended in Zambia. Itis important to improve our understanding of the reasons for poor uptake of HIV testing. However, the paucity of information would describe the extent of DCT service utilisation among TB patients in sub-Saharan ${ }^{23}$. Locally, Diagnostic Counselling and Testing (DCT) remains underutilised by patients diagnosed at Monze Mission Hospital. The percentage of patients with tuberculosis utilising the service was as low as 32.1percent. The percentage of TB patients who accepted HIV testing ranged between 26.1 and 43.4 percent from 2009 to $20142^{6}$. The figures are stump, indicating low utilisation of DCT uptake, despite being supported by appropriate policy guidelines and combined patient care provider education. However, there are still many challenges with regards to the integration of DCT activities.

The TB/HIV collaborative services Diagnostic Counselling and Testing (DCT) were introduced in 2004 in Zambia and initiated in 2008 at Monze Mission Hospital. DCT is being scaled up throughout the world. However, there is a paucity of information, and so little is known about the outcomes of DCT on subsequent behaviour. HIV testing and counselling should be offered to all Tuberculosis patients in settings where the HIV prevalence among TB patients exceeds 5 percent. Unfortunately, most of the programmes have been implemented vertically and therefore, have paid little attention to the linkages between TB and HIV infections ${ }^{26}$. These linkages were supposed to be coordinated by the TB/HIV working group of the Stop TB Partnership, which was established in 2001 to coordinate the global response to HIV associated TB epidemic.

Further, not many studies have been conducted on this topic in Zambia. The findings will be utilised to develop strategies that enhance DCT utilisation for HIV among patients with TB. The study will also assist in generating data that can be used as a basis for subsequent studies and investigations. The study will benefit many clients as they will be aware of the importance of HIV testing among tuberculosis patients is critical as it will help reduce morbidity and mortality rates because if found to be HIV positive, patients can access ART and continued care and support. DCT 
stands out as paramount to address the TB and HIV problems, make a new HIV diagnosis and get patients in care. DCT may benefit the patients as HIV will be diagnosed early, thereby preventing the dissemination of TB.

\section{MATERIALS AND METHODOLOGY}

\section{Population and sampling procedures}

Data stem from a hospital-based cross-sectional study at the Chest Clinic run at Monze Mission Hospital, Zambia. Monze is a second referral Hospital that treats and reviews patients with various diseases, including TB. The patients attend the clinic at appointed times advised by the Clinical Officer for continuous monitoring and consultation about their disease.

The sample size was calculated using SPSS Version 20.0 for windows computer software statistical package for data analysis. The sampling frame will comprise all TB patients visiting the chest clinic at the time of the study and meet the set criteria. The participants in the study will be selected using a simple random sampling method. Each individual within the population had a number and then used arbitrarily selected numbers from a table of numbers, giving each individual an equal chance to be selected for inclusion in the study. In this manner, a sufficient simple random sample of the general population became representative of the larger whole. According to Monze records 280 , the total population of TB patients that attend the clinic for one month is approximately at the research site. The participants will be identified and recruited consecutively for one (1) month to eliminate selection biases.

A total of about $280 \mathrm{~TB}$ patients are expected to pass through the chest clinic in one month. Thus, eight (8) participants will be selected randomly per day until the required sample size of 226 for the study is reached. The sample size was calculated basing on Krejcie and Morgan's ${ }^{18}$ formula for calculating sample size of a finite population. The calculated sample size comprised of 226 participants. A semi-structured interview schedule was used to capture data on demographic characteristics and factors results. The interview schedule was developed based on the World Health Organization (WHO) stepwise survey (STEPS) instrument ${ }^{22}$ ). The frequencies and descriptive statistics of the variables were calculated.

The relationships between the different categories of variables were investigated with Pearson's chi-square test for association and further fitting logistic regression model. The effects that levels of knowledge and confidentiality have on DCT utilisation were assessed with binary logistic regression. Only the independent variables that were determined to be significantly associated with the outcome variables after bivariate Chi-square testing was included in the logistic regression model. From the chi-squared association analysis, only the level of knowledge and confidentiality were statistically associated with DCT.

The study showed that the patients who did not utilise DCT had $47 \%$ decrease in the odds of achieving a high level of knowledge and confidentiality were less than 0.05 and therefore failed to reject the null hypothesis. Multivariate binary logistic regression model predicted that confidentiality and knowledge were associated with DCT at $\mathrm{p}$-value 0.05 . The Odds Ratio and 95\% confidence interval were calculated using binary logistic regression to identify DCT utilisation predictors while adjusting for confounders. A p-value of $<0.05$ was considered significant.

Inclusion criteria: Inclusion criteria were that all the confirmed tuberculosis out/in patients aged 1849 years were included in the study. The patients who were willing to participate in the study were asked to give informed and written consent. However, all diagnosed in/out TB patients were recruited and included in the study.

Exclusion criteria: TB patients outside Monze catchment referred to the chest clinic will not be included in the study because they may withdraw from the study due to distance. Patients aged less than 18 or more than 49 years who are referred to the ART sites for DCT will also not participate in the study. They may not consent to participate as they may think they are young or old to be at risk of contracting TB and HIV infection. Patients who will not consent or volunteer to participate in the study will be freely left out.

\section{RESULTS}

The data collected was entered and stored into the data editor of IBM ${ }^{\circledR}$ SPSS $®$ and statistically analysed using IBM ${ }^{\circledR}$ SPSS ${ }^{\circledR}$ Statistics for Windows 
Version 20.0. The data were subjected to double and consistency checks. This computer software statistical package enabled the researcher to obtain a data set of diagnostic counselling and testing and factors associated with utilisation. Frequency tables were used to present data and cross-tabulation was used to explore the statistical relationships between dependent and independent variables. The mean was applied to score ordinal variables such as levels of knowledge and education and staffing. The median was used to highlight the score at the exact centre of a frequency distribution. The relationships between the different categories of variables were investigated with Pearson's chisquare test for association and further fitting logistic regression model. The level of knowledge and confidentiality were statistically associated with DCT. Overall $(n=226)$, majority $150(66.3 \%)$ of the patients did not utilise diagnostic counselling and testing services while $76(33.6 \%)$ utilised diagnostic counselling and testing. The odds ratio of confidentiality was OR 0.52-1.637 and P-value 0.050 while the level of knowledge; OR was $0.719-45.785$, and P-value was 0.048 , and the variables were statistically significantly associated with Diagnostic Counselling and Testing The effects that levels of knowledge and confidentiality have on DCT utilisation were assessed with binary logistic regression. Only the independent variables that were determined to be significantly associated with the outcome variables after bivariate Chi-square testing was included in the logistic regression model. From the chi-squared association analysis, only the level of knowledge and confidentiality were statistically associated with DCT.

The study showed that the patients who did not utilise DCT had $47 \%$ decrease in the odds of achieving a high level of knowledge and confidentiality were less than 0.05 and therefore failed to reject the null hypothesis. Multivariate binary logistic regression model predicted that confidentiality and knowledge were associated with DCT at $p$-value 0.05 .

\section{DISCUSSION}

The current study showed that low DCT utilisation in most TB patients at Monze Mission Hospital was $l^{26}{ }^{26}$. Low DCT uptake has also been reported in other studies in developing and devel- oped countries. The possible explanation for this state of affairs might be inadequate knowledge on the importance of DCT uptake and confidentiality among things. On the other hand, interestingly, in studies conducted by ${ }^{18}$ in Alabama, high DCT utilisation was reported among patients. In another study done by Seyoum (2013) showed the association with low knowledge and DCT ${ }^{28}$. To the contrary, in Guyana, Malawi, and South Africa TB patients have higher literacy levels with consequent better knowledge levels about the relationship between TB/HIV/AIDS and its consequences ${ }^{27}$. In another, a study in Uganda finding that those with formal education were more likely to be offered a test ${ }^{2}, 5$ and another in Ethiopia showing that the most educated were less likely to be tested.

At Monze Mission Hospital, low utilisation of diagnostic counselling and testing service could be attributed to inadequate knowledge of the importance of DCT to rule out HIV and AIDS. The high DCT utilisation status is associated with confidence and knowledge in other studies because information, education and communication are effective ${ }^{20}$. The two variables found to be associated with DCT utilisation in the current study were the level of knowledge and confidentiality. This study showed that the patients who did not utilise DCT utilisation had 0.719-45.785 decreases in the odds of achieving a high level of knowledge compared to those who utilised DCT utilisation.

It is also not surprising that TB patients who fail to comply with DCT utilisation have low knowledge of the importance of testing, which results in poor outcomes ${ }^{21,30}$. The patients with low knowledge had odds ratio between 0.719-45.785 and P-value 0.048 .

However, in this study, less than half of those who utilised DCT had confidence in the health personnel while more than half had no confidence in the health personnel. In a study done by Fay and Glanz (2010) in San Francisco found that confidentiality was associated with non-utilisation of DCT. The odds ratio of patients with no confidence in the health personnel was 0.52-1.637, and P-value was 0.050 Tackling non-utilisation of DCT is not a simple matter, as it is multifactorial might include time, personality and patient-provider relationship ${ }^{21}$.In addition, this study showed that more than half of the patients were less likely to 
utilise diagnostic counselling and testing because they did not know the benefits of the test. These results agree with the findings by ${ }^{23}$ found that low rates of utilisation of DCT services were attributed to fear, stigma, discrimination, the timing of the service, and self-perceived risk of HIV infection among others ${ }^{29}$.

It is important to note that diagnostic counselling and testing ascertain the presence of HIV in TB patients as their chances of having HIV are high. Nonetheless, the testing of all TB patients is advantageous not only because it helps to detect HIV but also prevents complications that could arise if HIV is not detected early ${ }^{24,25}$. The study was carried out on a finite study population illustrated by the fact that only the participants who visited the Monze Mission Hospital-Chest clinic during the period of data collection were included in the sample. In order to achieve this, it is suggested that future research should focus on evaluating the effect of IEC on the utilisation of DCT among TB patients.

\section{Conclusion}

Diagnostic Counselling and Testing utilisation were low in the study population. Level of knowledge and confidentiality were the factors found to be associated with diagnostic testing and counselling. The findings suggest the need for information, education and communication as patients lack information on the importance of tuberculosis patients taking up the test. The majority of the patients in the current study did not utilise DCT. This was influenced by confidentiality and level of knowledge. Since tuberculosis like most other chronic diseases is progressive, the findings suggest that if the factors influencing low DCT utilisation are not adequately addressed, complications will arise and drug therapy becomes much more complex with time. The study will enable health care providers to provide information, education and communication to the patients to ensure high DCT utilisation.

\section{Acknowledgements}

I would like to thank the participants for their role in providing valuable data for the successful completion of the study at no cost.

\section{Conflict of interest}

The authors declare no competing interests.

\section{Ethical Clearance}

This study was approved by ERES Converge (Reference number2014-AUG-019).

\section{REFERENCES}

1. Mutanga JN, Raymond J, Towle MS, Mutembo S, Fubisha RC, Mutembo S, et al. institutionalising provider-initiated HIV testing and counselling for children. An observational case study from Zambia 2012; 23:32-34.

2. WanyenzeRK, Nawavvu C, Namale AS, Alice S, and Manyanja B. Acceptability of routine HIV counselling and testing and HIVsero-prevalence in Uganda. Bull World Health Organ, 2008; 86:- 302-9,http//www. ncbi.nm.nih.gov/NCBI/Literature/PubMed Central 2647415 102471/BLT.07.042580 accessed on 2/04/13 at 18:00 hours.

3. World Health Organization. Global Tuberculosis Control Epidemiology Strategy Financing; 2009; Geneva.

4. UNAIDS. (, 2012). Global Report on AIDS information; Geneva.

5. Moore D, Liechty C, Edward P, Were W, and Mina G. Prevalence, incidence and mortality associated with tuberculosis in HIV-infected patients initiating antiretroviral therapy2007; 21:713-719 http//journals.plos.org/plosone/ article/id 10.1371/journal.pone.0111209 accessed on 18/10/14.

6. Affusim CC. Kisame BE, and Abah VO, The pattern of presentation and prevalence of tuberculosis in HIV-seropositive patients in Nigeria 2012; 112-125.http//www.hindawi. com/journals/isrn/2012/326572 accessed on $21 / 08 / 13$ at $15: 00$ hours.

7. UNDP (2013). The rise of South Africa Africa, Human Development Report; 43; Geneva.

8. Pawlowski A, Jansson M, Skold M,Rottenberg ME, and Kallenius G, Tuberculosis and HIV co-Infection, PLoS Pathogens 2012; 8:1-7. http//repository.usu.ac.id/bitstream/.../41182/ accessed on 16:07 hours. 
9. Markowitz N, Hansen NI, Hopewell PC, Glassroth J, Kvale PA, Incidence of Tuberculosis in the United States among HIV-infected Persons. Ann Intern Med 1997; 126:123-32.PMID: 9005746; https://biolincc. nhlbi.nih.gov/static/studies/pacs/\%20 PACs\%20Publications.pdf accessed on 12/03/15.

10. Ghiya R, Naik E, Casanas B, Izurieta R, and Marfatia YC, Epidemiological profile of HIV/TB co-infected patients in Vadodara, Gujarat, Indian J Sex Transm Dis 2009; 30:10-5.Journal of Evolution of Medical and Dental Sciences/ Volume 2/ Issue 21/ May 27, 2013. http//dx.doi.org/10.14260/jemds/759 accessed on 30/4/15.

11. Watch A, Kuttiatt VS, Sharma, A, Singh S, and Varma S, CD4 cell count recovery in HIV/TB infected patients versus uninfected HIV patients, Indian J Pathol Microbial 2010 ; 53(4):745-9. Indian journal of pathology \& microbiology 2010; 53(4):7459. 2010: Surjit Singh ...http//docbydoc.com/ Profile/1314172 accessed on 10/5/15.

12. Bhagyabati DS, Santa N, Jeetenkumar ST, Singh TI, Singh KB, Prasad L, et al. The HIV and TB co-infection JIACM; 2005; 6 (3):220-3. Journal, Indian Academy of Clinical Medicine http//www.ijmsph. $\mathrm{com} /$ ?mno $=47783$ accessed on $23 / 01 / 15$ at 20:09 hours.

13. Ministry of Health (2009). TB/HIV Guidelines, Lusaka, Zambia.

14. World Health Organization (2007). Guidance on Provider Initiated HIV Testing and Counseling in Health Facilities, Geneva, Switzerland.

15. World Health Organization (2014). TB/HIV Guidelines, Geneva.

16. Chimzizi R, Bwanali, A. Mbalume, D, Teck R, Gomani P, Zachariah R, et al. Voluntary Counselling, HIV Testing and Adjunctive Cotrimoxazole are associated with improved TB treatment outcomes under routine conditions, PMID: Tuberc Lung Dis, 2004.http//www.ncbi.nlm.nih.gov/ pubmed/1137534 accessed on $09 / 03 / 14$ at 20:32hours.
17. Krejcie RV, and Morgan DW, Determining sample size for research activities. Educational and Psychological Measurement. The published formula for determining sample size. He relationship between sample size and total population. Accessed on 6/04/2014 at 17:39 hoursfromhttp//home.kku.ac.th/ sompong/quest speaker/Krejcie and Morgan article pdf, 1970; 30:607-610.

18. Yang $\mathrm{C}$, Chheng $\mathrm{P}$, kimerling $\mathrm{ME}$, and Song $\mathrm{N}$, Improving integrated care for HIV positive patients with TB in Alabama, 2007;85 (5): 382-383.http//www.the union.org/what-wedo/journals/ijtld/.../Abstract-Book-2007 accessed on 29/03/2014 at 12:45 hours.

19. Santos ET, What helps, What hurts: Client and frontline provider perspectives on $\mathrm{TB} /$ HIV interaction; beyond pills; How to support clients in TB and HIV treatment, Stop TB Coordinating Board, Geneva. http//www. theunion.org/what-we-do/journals/ijtld/.../ Abstract-Book-2007 accessed on 14/02/2013 at 09:10hours.

20. Check date values in: |accessdate $=($ help$)$ Deribew A, Negussue N, Kassahun W, Apers $\mathrm{L}$, andColebundersR, uptake of providerinitiated counselling and testing among TB suspects. Department of Epidemiology, Jimma University, Ethiopia, PMID; 2009: $20937185 \mathrm{http} / / \mathrm{www} . n c b i . n l m . n i h . g o v /$ pubmed/20937185 accessed on 14/03/14 at 14:00 hours.

21. Glanz K, and Donald B, The Role of behavioural science theory in developing and implementing Public Health Intervention; Annual Review of Public Health; 2010; 31: 399-418.http//www.med.upenn.edu/chbr/.../ glanz-BishopARPH31_399-418_2010.pdf accessed on 28/09/13 at 10:39 hours.

22. Kipp AM, Pungrassami P, Nilmanat K, et al. Socio-Demographic and AIDS-related factors associated with Tuberculosis stigma in Southern Thailand, University of North Caroline, U.S.A. 2011, 30:11:675.http// www.ncbi.nlm.nih.gov/pubmed/21878102 accessed on 26/07/13 at 21.00hours. 
23. Nateniyom S, Jittimanee SX, and ViriyakitjarD, Provider initiated diagnostic HIV counselling and testing in tuberculosis clinics in Thailand. Bureau of Tuberculosis, Department of Disease Control, Int J Tuberc Lung Dis, Thailand 2008; 12(8):955961, http//www.ncbi.nlm.nih.gov/ pubmed/20937185 accessed on 19/04/14 at 09:39hours.

24. David C, Katherine H, and Eric G, Integrating tuberculosis and HIV care in the primary care setting, Tropical Medicine and International Health, South Africa, 2004; 9(6): A11A15Supplement.Vol.9 no. 6 pp.a11-a15.38. Ibid.39.https//qspace.library.queensu. ca/.../Houston_Adam_R_201101_MA.pdf accessed on $14 / 11 / 13$ at $14: 18$ hours

25. World Health Organization. Global Tuberculosis Control Epidemiology Strategy Financing, WHO Report, Geneva, Switzerland: WHO; 2009.

26. Monze Mission Hospital. Tuberculosis Reporting Form. 2011, Monze.
27. Gasana M, Vandebriel G, Kabanda G, and Tsouris SJ, Integrating tuberculosis and HIV care, Int $\mathrm{j}$ Tuberc Lung Dis, 2008; 12:3943. http//www.ncbi.nlm.nih.gov/pubmed 18302821 accessed on $13 / 07 / 14$ at $22: 40$ hours.

28. Seyoum A, and Legesse M, (2013). HIV and perception about diagnostic counselling and testing among TB patients, 2013; PMCID: PMC3600624, 10.http//www.biomedcentral. com/1471-2458/13/124 accessed on $28 / 05 / 2014$ at $15: 34$ hours.

29. Kigozi NG, HeurisJC, and Wouters, Tuberculosis patients reasons for and suggestions to address non-uptake of HIV testing, Free State, South Africa, Centre for Health Systems Research and Development, 2008; PMCID: PMC3112395675http// www.ncbi.nlm.nih.gov/pubmed/21599883 accessed on $26 / 07 / 14$ at 21.00 hours. 
Table 1: The demographic characteristics of participants that utilised and did not utilise DCT are shown in Table 1

\begin{tabular}{|l|c|c|c|}
\hline \multicolumn{5}{|c|}{ DCT utilisation } \\
\hline Characteristic & Utilised (Yes) & Did not utilise (No) & P-Value* $^{*}$ \\
\hline Age & No. (\%) & No. (\%) & \\
\hline $18-25$ years & & & \\
\hline $26-33$ years & $8(17.0)$ & $39(82.9)$ & \\
\hline $34-41$ years & $21(35.5)$ & $38(64.4)$ & 0.773 \\
\hline $42-49$ years & $21(33.3)$ & $42(66.7)$ & \\
\hline Sex & $22(33.3$ & $44(66.7)$ & \\
\hline Male & & & \\
\hline Female & $48(38.4)$ & $77(61.6)$ & \\
\hline Education level & $28(27.7)$ & $73(72.6)$ & 0.160 \\
\hline Primary & & & \\
\hline Secondary & $31(39.2)$ & $48(60.7)$ & \\
\hline College & $32(28.3)$ & $81(71.7)$ & 0.970 \\
\hline University & $12(50.0)$ & $12(50.0)$ & \\
\hline None & $0(0.0)$ & $4(100)$ & \\
\hline Marital status & $1(20)$ & $4(80)$ & \\
\hline Single & & & \\
\hline Married & $26(36.1)$ & $46(63.9)$ & \\
\hline Separated & $31(29.2)$ & $75(70.8)$ & 0.388 \\
\hline Divorced & $9(52.9)$ & $8(47.1)$ & \\
\hline Widowed & $4(28.6)$ & $10(71.4)$ & \\
\hline & $6(35.3)$ & $11(64.7)$ & \\
\hline
\end{tabular}

Table 2: Diagnostic Counselling and Testing utilisation by the Determinant Factors

\begin{tabular}{|l|c|c|c|}
\hline & \multicolumn{1}{|l|}{ DCT utilisation } & \multicolumn{1}{l|}{ Low } & P-Value* \\
\hline & No (\%) & No (\%) & \\
\hline Characteristics & & & \\
\hline Staffing & $3(12.5)$ & $21(87.5)$ & \\
\hline Poor & $29(40.8)$ & $42(59.1)$ & \\
\hline Moderate & $43(43.4)$ & $56(56.6)$ & \\
\hline Adequate & \multicolumn{3}{|l|}{} \\
\hline Waiting time & $36(40.9)$ & $36(40.9)$ & \\
\hline Short & $40(22.5)$ & $40(22.5)$ & \\
\hline Long & & & \\
\hline Level of knowledge & $27(36.5)$ & $47(63.5)$ & \\
\hline High & $1(8.3)$ & $11(91.7)$ & $\mathbf{0 . 0 4 5}$ \\
\hline Medium & $48(34.3)$ & $92(65.7)$ & \\
\hline Low & & & \\
\hline Confidentiality & $31(34.4)$ & $59(65.5)$ & \\
\hline Yes & $44(32.8)$ & $90(67.2)$ & $\mathbf{0 . 0 4 9}$ \\
\hline No & & & 0.803 \\
\hline Stigma & $31(41.3)$ & $44(58.7)$ & \\
\hline Stigmatised & $44(32.8)$ & $90(67.2)$ & \\
\hline Not stigmatised & & & \\
\hline Time of the service & $45(59.2)$ & $31(40.8)$ & 0.515 \\
\hline Good & $82(54.7)$ & $68(45.3)$ & \\
\hline Poor & & & \\
\hline Attitude & $107(71.8)$ & $42(28.2)$ & \\
\hline Negative & $50(65.8)$ & $26(34.2)$ & \\
\hline Positive & & & \\
\hline & & & \\
\hline
\end{tabular}


Table 3: Multivariate Binary Logistic Regression Model of determinant Factors Associated with Diagnostic Counseling and Testing

\begin{tabular}{|l|c|c|c|c|}
\hline & DCT & & \\
\hline & High & Low & & \\
\hline Predictor Variable & No (\%) & No (\%) & AOR (95\% CI) & P-Value* \\
\hline Knowledge & $27(36.5)$ & $47(63.5)$ & $0.90(0.504-1.635)$ & 0.099 \\
\hline High & $1(8.3)$ & $11(91.7)$ & $5.73(0.719-45.785$ & $\mathbf{0 . 0 4 8}$ \\
\hline Moderate & $48(34.3)$ & $92(65.7)$ & $1.00($ Ref) & \\
\hline Low & & & $0.93(0.52-1.637)$ & $\mathbf{0 . 0 5 0}$ \\
\hline Confidentiality & $31(34.4)$ & $59(65.6)$ & $1.00($ Ref) & \\
\hline Yes & $44(32.8)$ & $90(67.2)$ & & \\
\hline No &
\end{tabular}

